\title{
Sleep Disorders and Cancer
}

\author{
Miguel Ángel Martínez-García ${ }^{1,2}$ • Francisco Campos-Rodríguez ${ }^{3}$ - Isaac Almendros ${ }^{4,5}$
}

Published online: 11 February 2016

(C) Springer International Publishing AG 2016

\begin{abstract}
There is a growing evidence that sleep disorders are associated with an increased incidence and accelerated cancer progression. In the light of the previously established relationship between hypoxemia and cancer, obstructive sleep apnea and its main consequence, intermittent hypoxia, could be associated with a poorer prognosis or higher incidence of cancer. From this pathophysiological starting point, and in the light of animal studies that confirmed this association, two separate research groups observed in large-scale clinical and epidemiological series that the degree of nighttime hypoxia found in patients with obstructive sleep apnea was associated with a higher incidence of cancer, as well as a higher mortality rate although their retrospective nature means that they should be considered with caution. A study currently in progress with a large group of patients with melanoma may soon cast new light on the existence or not of this association and its underlying pathophysiological mechanisms.
\end{abstract}

Keywords Sleep-disordered breathing · Cancer · Sleep apnea $\cdot$ Intermittent hypoxia $\cdot$ Sleep duration $\cdot$ Melatonin . Shift work

Miguel Ángel Martínez-García

mianmartinezgarcia@gmail.com

1 Pulmonology Service, University and Polytechnic La Fe Hospital, Valencia, Spain

2 Servicio de Neumología, Hospital Universitario y Politécnico La Fe, Bulevar Sur s/n, 46016 Valencia, Spain

3 Pulmonology Service, Valme University Hospital, Seville, Spain

4 Biophysics and Bioengineering Unit. Facultad de Medicina, Universidad de Barcelona, Barcelona, Spain

5 CIBER de Enfermedades Respiratorias (CIBERES), Madrid, Spain

\section{Introduction}

There is a growing evidence of an association between abnormal sleep and the incidence and progression of cancer. Shift work involving the disruption of circadian rhythms has been shown to increase carcinogenesis in animal models. Suppression of melatonin induced by exposure to light at night, coupled with deregulation of clock genes, may potentially contribute to the promotion and induction of cancer, particularly in endocrine tissues. This evidence has led the International Agency for Research on Cancer to classify shift work with circadian disruption as "probably carcinogenic." As regards sleep duration, its relationship with cancer is less clear and various studies have yielded contradictory findings. Finally, the relationship between obstructive sleep apnea (OSA) and cancer opens up an exciting field of research for the coming years, although at the moment, clinically speaking, it stands on unsteady ground. Nevertheless, both the pathophysiological and animal studies undertaken to date seem to make this association more credible. From a clinical viewpoint, several studies have observed an association between OSA (particularly when evaluated in terms of the resulting hypoxemia) and the incidence of and mortality from cancer, although their retrospective methodology means that further research is needed before any more unequivocal conclusion can be reached. All the indicators seem to suggest that if such an association were to exist, it would be one of the most important discoveries so far in the field of sleep apnea research, as both OSA and cancer share certain common epidemiological characteristics such as high prevalence, a major healthcare impact and a potential treatability. The following review seeks to inform readers of the findings to date on the relationship between sleep disorders, especially OSA and cancer, as well as review the potential pathophysiological mechanisms that would support the plausibility of the hypothesis 
that sleep disorders might affect carcinogenesis or cancer progression.

\section{Pathophysiological Aspects}

There are several pathophysiological mechanisms that could give biological plausibility to a possible relationship between some sleep disorders including OSA and either the conversion of healthy cells into malignant ones or the spread and growth of a tumor. The main mechanisms or biomolecular pathways that are potentially involved are discussed below.

\section{Melatonin}

In addition to its regulatory role of the sleep circadian cycle, melatonin has been shown to inhibit cancer development and growth and improve immune function. This anticancer activity of melatonin involves antimitotic activity, resulting from melatonin's direct effect on hormone-dependent proliferation. Melatonin suppression in shift workers through exposure to light at night can result in an increased release of gonadotropins from the pituitary gland, thereby stimulating hormones such as testosterone or estrogen $[1,2]$. Melatonin also neutralizes and removes free radicals and has a protective effect against the DNA-damaging effects of hydrogen peroxide [3]. It inhibits tumor signal transduction and the metabolic activity of cancer cells via the activation of melatonin receptor MT1 and increases the expression of the tumor-suppressor gene p53 [4]. Blunted melatonin secretion may also lead to immune suppression with potential consequences on the development and growth of cancer [1].

\section{Clock Genes}

Clock genes play a major role in circadian synchronization and phase shift, but also seem to be important in tumor suppression and promotion $[2,3]$. Clock genes regulate cell proliferation and apoptosis by controlling a number of cell cyclerelated genes, cell cycle checkpoints, and tumor-suppressor genes that mediate response to DNA damage. Chronic exposure to shift work may disrupt circadian rhythms and stimulate tumor development and progression, partly mediated by epigenetic changes [2, 3, 5-10].

\section{Oxidative Stress}

The pattern of desaturation and reoxygenation that defines intermittent hypoxia is a characteristic of OSA and represents a major stimulus to the activation of the oxidative stress system through the production of reactive oxygen species (ROS) $[11,12]$. This leads to an imbalance between the production and degradation of certain oxidants and antioxidants. This phenomenon has been related to an increase in both acute and chronic mutagenesis, various alterations in cellular function and structure, damage to DNA, and instability in the genome, which can result in greater cellular proliferation and neoplastic transformation. Lastly, oxidative stress has been related to transcription factors such as activating protein 1 (AP-1) and nuclear factor $\mathrm{kB}(\mathrm{NF}-\mathrm{kB})$, which are involved in a greater propensity to develop cancer [13].

\section{Hypoxia-Induced Factor (HIF-1)}

All the cell types have various compensatory mechanisms in response to hypoxia, whether continuous or intermittent (as in OSA). One of the most powerful mechanisms is the increased production of a key molecule known as hypoxia-induced factor (HIF-1) (Fig. 1). HIF-1 orchestrates the regulation of genes that codify mediators allowing cells to adapt to situations of tissue hypoxia. HIF-1 is made up of two subunits: HIF-1 $\alpha$ and HIF- $1 \beta$, the former being the most important molecule in the regulation of tissue hypoxia [14•]. Hypoxic compensation occurs when HIF-1 $\alpha$ sets in motion a series of mechanisms that activate the production of angiogenic citokines, most notably the vascular endothelial growth factor (VEGF), which regulates the formation of new collateral blood vessels that supply the hypoxic area with more oxygen and avoid areas with vascular obstruction [15-21]. Although this process is an important compensatory mechanism in cardiovascular diseases with associated tissue ischemia, it seems to have a damaging effect in cancer patients. Large hypoxic areas (with $\mathrm{pO}_{2}$ below $10 \mathrm{mmHg}$ ) can be found inside tumors, and these are powerful activators of the compensation systems mediated by HIF-1. When this chronic hypoxia that frequently characterizes solid tumors is aggravated by the intermittent hypoxia that occurs in cancer patients with OSA, the resulting tumor neovascularization may therefore provide a greater opportunity for cancer cells to reach the circulatory system an metastasize to other organs. Furthermore, this neovascularization occurs at the expense of fragile, anomalous vessels and so the reoxygenation is far from optimal.

\section{Systemic Inflammation}

There is an increase in both local and systemic inflammation in OSA [22, 23]. The role of OSA as a cause of systemic inflammation is the most important aspect of the possible association between cancer and OSA. As noted above, an oxidant/antioxidant imbalance and an increase in ROS have been associated with a systemic increase in the concentration of pro-inflammatory substances, including the tumoral necrosis factor (TNF- $\alpha$ ), interleukin (IL)-6, and IL- 8 , via the abovementioned activation of transcription factors NF-kB and AP1. The NF-kB factor is considered to play a key role in the transcription of many genes related to inflammation, 
Fig. 1 Pathophysiological relationship between obstructive sleep apnea and cancer. Obesity as a confounding factor. $H I F-1$ hypoxia inducible factor-1, $V E F G$ vascular endothelial growing factor, $R O S$ reactive oxygen species

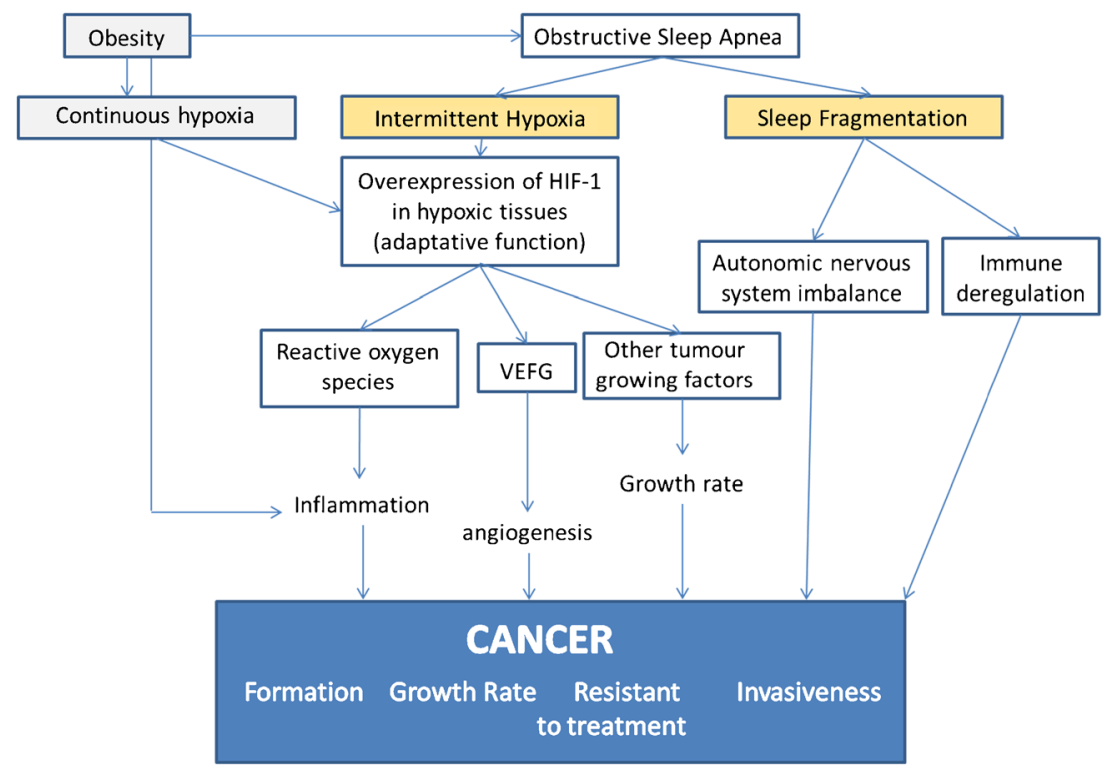

atherosclerosis, and cancer. Intermittent hypoxemia seems to be the most important element in the activation of this inflammatory element in patients with OSA [22, 23].

The inflammation associated with obesity, which could be described as chronic low-degree inflammation created by adipocytes in response to an excess of calories and nutrients, seems to be an important element in this relationship and could be independent of the existence of OSA since the prevalence of obesity in patients with OSA is abnormally high [24] and obesity has been associated with increased risk of some types of tumors [25-28].

\section{Immune System}

Macrophages are the most abundant component of tumor stroma and play an important role in most of malignant processes. These cells can modify their phenotype from proinflammatory or classically activated macrophages (M1) to an alternative activated form (M2), characterized by expression of angiogenic and tissue remodeling molecules [29]. Application of intermittent hypoxia (IH) can increase the number of macrophages in some tissues such as adipose and aortic walls. Those macrophages $\mathrm{IH}$-induced macrophages present a shift toward classical activation phenotype which has been linked to the metabolic and cardiovascular consequences observed in OSA patients [30,31]. The increased number of macrophages can result from an accelerated proliferation of the preexisting resident macrophages as well as an increased release of bone marrow-derived monocytes [32]. In terms of cancer, inflamed macrophages within the adipose tissue surrounding the tumor have been proposed as a depot of macrophages to the tumor [33]. Although the M1 phenotype induced by $\mathrm{IH}$ is associated to tumor surveillance, later has been shown that $\mathrm{IH}$ not only promoted increased number of resident and bone marrow macrophages in the adipose tissue but also accelerated reeducation toward M2 in the tumor boundaries and increase their recruitment [34].

Recent research has shown that alterations in the immune response induced by IH and SF can mediate in part the accelerated tumor progression observed during both conditions. Almendros et al. revealed that IH increased the recruitment of tumor-associated macrophages, lymphocyte $\mathrm{T}$ regulatory cells (Tregs) and myeloid-derived stem cells to the tumor [35].

\section{Evidence from Animal Models}

\section{Animal Models on Circadian Disruption and Cancer}

In support of the hypothesis of a causal relation between shift work and cancer, there is a strong evidence from animal models that repeated disruption of the circadian rhythms, dysregulation of some of the clock genes, and experimental melatonin-suppressing manipulations promote the development and growth of malignancies.

Shah et al. [36] exposed female rats to constant light immediately after birth, provoking melatonin deprivation. The loss of inhibitory regulatory control over the hypothalamichypophysis axis led to constant availability of estrogen and increased the risk of mammary cancer. Administration of melatonin reduced cancer incidence. Blask et al. [37] showed that exposure of rats bearing human breast cancer xenografts to increasing intensities of white fluorescent light during each 12-h dark phase resulted in a dose-dependent suppression of nocturnal melatonin blood levels and a stimulation of tumor growth. When these tumors were perfused with physiologically melatonin-rich blood collected from healthy, premenopausal female volunteers during the night, the proliferative activity 
was largely suppressed, suggesting that tumor growth response to exposure to light during darkness is intensity dependent.

Filipsky et al. [38] tested the effect of functional disruption of the circadian system on tumor progression in an experimental model of chronic jet lag. B6D2 $\mathrm{F}_{1}$ mice were synchronized with $12 \mathrm{~h}$ of light and $12 \mathrm{~h}$ of darkness or underwent repeated 8 -h advances of the light/dark cycle every 2 days before inoculation with Glasgow osteosarcoma. The authors observed that the tumor grew faster in the jet-lagged animals as compared to controls, whereas exposure to constant light or darkness had no effect. Whereas the expression of mPer2 mRNAs showed significant circadian rhythms in controls, these were suppressed in jet-lagged mice. The same authors conducted another study in which the suprachiasmatic nuclei of mice were destroyed by bilateral electrolytic lesions and Glasgow osteosarcoma or pancreatic adenocarcinoma was inoculated [39]. Disruption of circadian rhythms was confirmed by measuring body activity, temperature, and plasma corticosterone levels. Both types of tumors grew two to three times faster in mice with destroyed suprachiasmatic nuclei compared to sham-operated mice.

\section{Animal Models on OSA and Cancer}

\section{Intermittent hypoxia and sleep fragmentation mimicking OSA accelerate tumor growth and invasiveness}

The potential effects of intermittent hypoxia (IH) and sleep fragmentation (SF) on tumor progression have been investigated recently in some types of cancer. Almendros et al. showed that melanoma increased tumor growth, metastasis, and mortality in mice subjected to $\mathrm{IH}[40,41 \bullet \bullet, 42]$. Mice were subjected to $\mathrm{IH}$ consisting in cycles of $20 \mathrm{~s}$ of hypoxia $\left(5 \% \mathrm{FiO}_{2}\right)$ followed by $40 \mathrm{~s}$ of normoxia $\left(21 \% \mathrm{FiO}_{2}\right) 6 \mathrm{~h} /$ day during 14 days to study tumor growth or 28 days for mortality and incidence of metastasis. The results from those studies showed that IH double increased tumor size as well as promotes a marked increase in number and metastatic area in the lungs $[40,41 \bullet \bullet$. Later, a similar model that used lung carcinoma was employed to test tumor growth and malignancy in response to ether $\mathrm{IH}\left(90 \mathrm{~s}\right.$ hypoxia $\left(6 \% \mathrm{FiO}_{2}\right)$ followed by 90 s normoxia (21\% FiO2)) 12 h/day during 28 days [34, 35]. As occurred in melanoma tumors, lung carcinoma experienced increased growth and invasiveness toward adjacent tissues in response to both paradigms.

Models using SF showed similar results than those using IH. Application of SF during 6 weeks in male mice was able to increase lung carcinoma growth with increased number of TAMs in those peripheral areas where invasion process occurs [43]. In this work, Hakim et al. proposed that the cancer malignancy induced by SF could be mediated in part through TLR4 receptors and their downstream pathways mediated by MyD88 and TRIF molecules. In addition, an increased expression of metalloproteinase 9, a well-known molecule participating in extracellular matrix degradation, was observed overexpressed by intravital microscopy in SF condition in vivo. Although another type of tumors still needs to be evaluated, the data from animal models support the notion that both the IH and SF can enhance tumor aggressiveness.

\section{Evidence in Humans}

\section{Shift Work and Cancer}

Evidence of an association between chronic circadian disruption and cancer in humans is more limited than that derived from animal models. Several epidemiological studies have revealed, however, that human night shift workers might be at an increased risk of cancer. Against this background of laboratory animal and human findings, the World Health Organization International Agency for Research on Cancer (IARC) concluded in 2007 that shift work involving circadian disruption is probably carcinogenic in humans [44].

Given that melatonin is one of the most likely mechanisms involved in this association, most human research has focused on endocrine cancers such as breast, prostate, and endometrial tumors. Schernhammer et al. [45] prospectively studied the relationship between rotating night shift work and breast cancer risk in 115,022 premenopausal nurses. After 12 years of follow-up, the risk of breast cancer was found to be slightly higher in women who worked for 20 or more years on rotating night shifts, compared with those who never did so (multivariate RR 1.79; $95 \%$ CI 1.06-3.01). In a similar study, Viswanathan et al. [46] reported that women who worked for 20 or more years on rotating night shifts had a significantly increased risk of endometrial cancer (RR, 1.47; $95 \%$ CI 1.03 to 1.14$)$ and that total years working on rotating night shifts was associated with an increased endometrial cancer risk $(P$ trend $=0.04$ ).

Hansen et al. [47] compared 7035 women aged $30-54$ years with a diagnosis of breast cancer with a similar number of matched controls and obtained similar results. After accounting for confounders, they found a 1.5-fold increase in the risk (95\% CI, 1.2 to 1.7 ) of primary breast cancer in women who had worked for least half a year in any trade with predominantly night work. A trend was observed of risk increasing in accordance with increased duration of nighttime employment.

A study in Finland of 10,935 women with visual impairment showed an inverse association between breast cancer incidence and the degree of visual impairment, suggesting a dose-response relationship between visible light (and thus a very probable involvement of melatonin) and the risk of breast cancer [48]. 
This association has also been shown in males. Kubo et al. [49] prospectively examined the association between shift work and the risk of prostate cancer incidence in 14,052 working men in Japan. Compared with day workers, rotating shift workers were significantly more at risk of prostate cancer (RR $3.0,95 \%$ CI 1.2 to 7.7 ).

\section{Sleep Duration and Cancer}

Although some studies have found that short sleep duration is related to an increased risk of some types of cancer, particularly breast cancer, the data are controversial and other studies have neither reported any association nor even shown any association between long sleep duration and cancer [50-57]. Two main reasons may explain these disparate findings. Most studies were based on self-reported sleep duration instead of objective measures such as actigraphy or polysomnography. Additionally, sleep duration is usually measured only once, at baseline, but in long-term follow-up studies sleep, duration is likely to change throughout the follow-up, which may also influence the outcomes. Table 1 depicts the results of the most important studies to address this association.

\section{Sleep Apnea and Cancer}

Unfortunately, very few studies to date have analyzed the possible association between OSA and cancer in humans (Table 2).

\section{Population-Based Studies}

The first population-based study to analyze the association between OSA and cancer was the Wisconsin cohort, comprising 1522 subjects followed up for 22 years [58••]. This study found a dose-response association between the severity of OSA, as measured by the apneas-hypopnea index (AHI), and total cancer mortality. This association remained after adjusting for various confounders, and severe OSA $(\mathrm{AHI} \geq 30)$ even proved to be an independent predictor of mortality from cancer. This association was even stronger when an oxymetric index, the percentage of nighttime sleep with oxygen saturation $<90 \%$ (Tsat $90 \%$ ), was used instead of the AHI as a marker of the severity of OSA. This association was stronger in non-obese patients.

Furthermore, the Busselton cohort, comprising 400 subjects followed up for 20 years, also found an association between moderate-severe OSA ( $\mathrm{AHI} \geq 15)$ and greater mortality (HR 3.4, $95 \%$ CI 1.1-10.2) and cancer incidence (HR 2.5, $95 \%$ CI 1.2-5.0) [59].

Finally, two large Taiwanese series [60, 61], using data from their National Health Institute, observed that OSA patients had a greater risk of developing breast cancer (HR 2.09; $95 \%$ CI 1.06-4.12) [60] or a primitive cancer of the central nervous system (HR 1.54; $95 \%$ CI 1.01-2.37) [61], compared to control groups with no OSA. Both these studies presented significant limitations, however, such as a lack of data on the severity of OSA and of any adjustment for several basic confounding variables.

One study that used symptoms as markers of OSA did not find any association between the presence of snoring, witnessed pauses or excessive daytime sleepiness, and a greater incidence of cancer [65]. This association, however, may have been underestimated since the participants did not undergo any objective sleep tests to confirm or rule out the presence of OSA.

\section{Clinical Studies}

In 2013, the Spanish Sleep Group published the first clinical study to analyze the association between OSA and cancer. This was a retrospective, multicenter study with a mean follow-up of 4.5 years. An initial analysis studied the association between the severity of OSA and the incidence of any type of cancer in 4910 patients without previous cancer [62・•]. After adjusting for various possible confounders, a significant association was found between the incidence of cancer and the severity of OSA as measured by the Tsat $90 \%$, but not as measured by the AHI. The strongest association was found in the group of patients $<65$ years, where the incidence of cancer was related to both the AHI and the Tsat $90 \%$. When the mortality in this series was analyzed, the results were very similar to those obtained for incidence, with a significant association between mortality from cancer and Tsat $90 \%$ as a marker of the severity of OSA, particularly in subjects aged under 65 years [63]. A sub-analysis including only patients with cancer $(n=527)$ found that Tsat $90 \%$ was an independent predictor of cancer lethality.

These results were not reproduced, however, in another very recent study in Toronto that analyzed 10,149 patients with suspicion of OSA between 1994 and 2010, with a follow-up of 7.8 years [64]. After adjusting for several variables, the authors did not find any greater incidence of cancer in patients with severe OSA measured by either AHI or Tsat $90 \%$, compared to patients with no OSA (AHI $>30$ vs. $<5$ : adjusted HR 1.02, $95 \%$ CI 0.80-1.31; Tsat90 \%: adjusted HR $1.00,95 \%$ CI $0.99-1.02$ ). These results did not vary when the authors used the same cutoff points for AHI and Tsat $90 \%$ as the Spanish Sleep Group Study.

Although five of the seven studies carried out to date have found an association between the severity of OSA, especially when measured by oxymetric markers, and cancer incidence

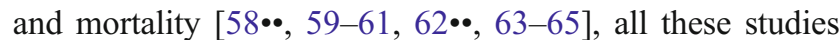
present several limitations that oblige us to approach their conclusions with caution and continue researching this association. None of the series were specifically designed to analyze the association between OSA and cancer; most of them 


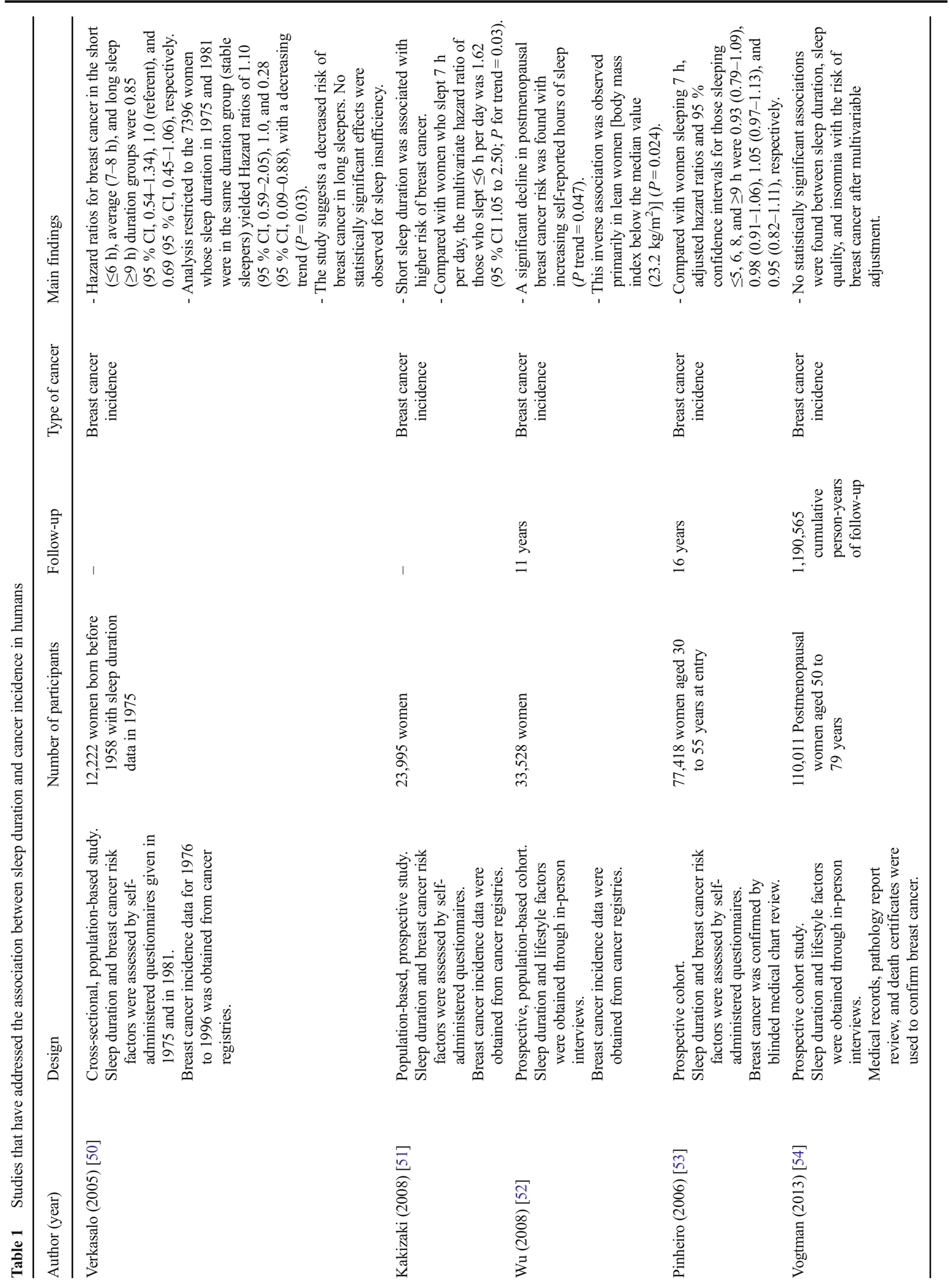




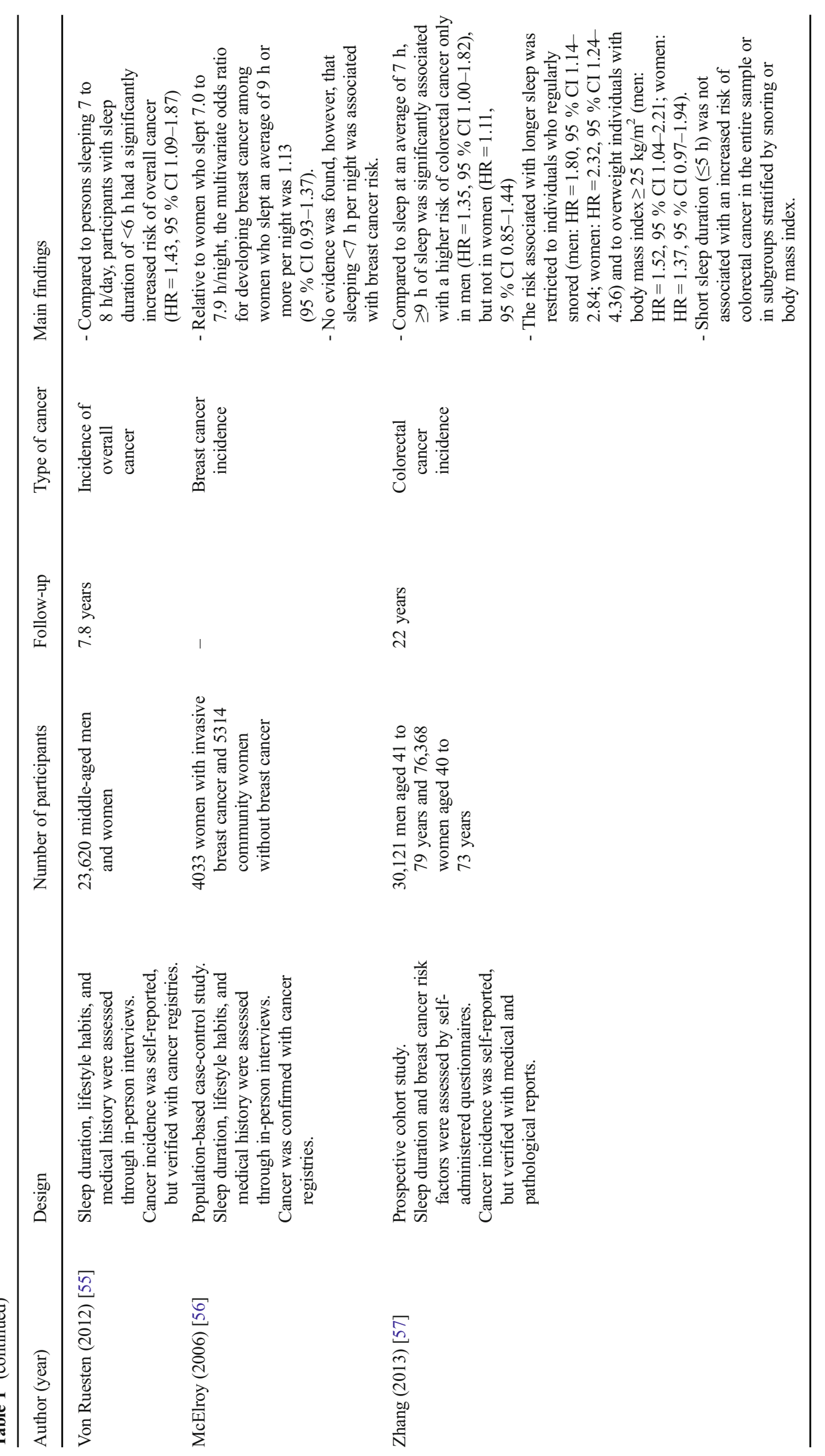




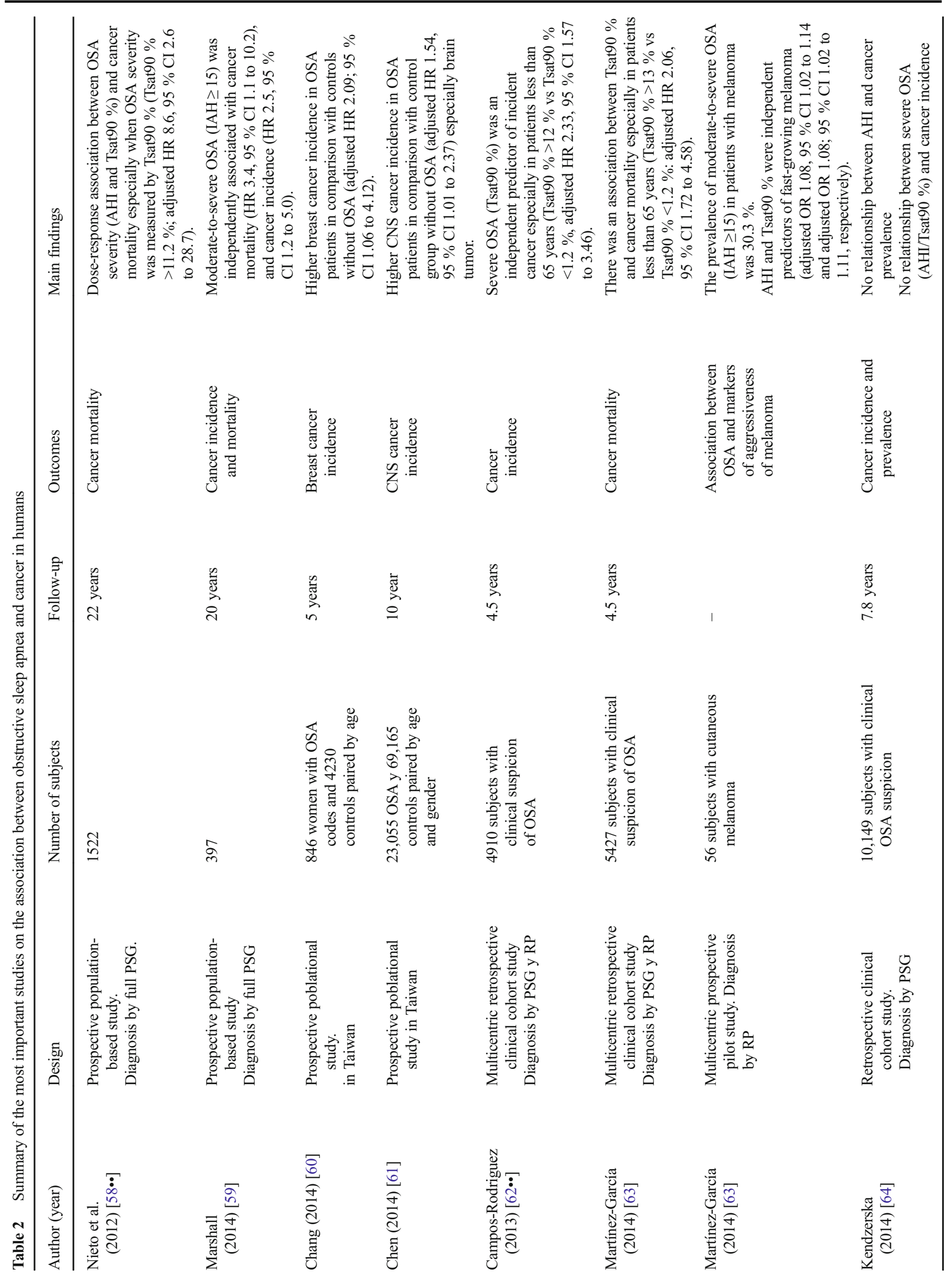


Table 3 Future research challenges on the relationship between sleep apnea and cancer

To identify which cancer types have more robust association with intermittent hypoxia and sleep fragmentation

To further analyze the role of the possible confounders especially obesity

To identify high-risk groups related to age, gender, diurnal hypersomnia or the presence of obesity

To identify the main pathophysiological pathways

To establish which polysomnographic variables better predict cancer risk

To analyze the role of CPAP treatment on modifying the association between OSA and cancer risk

To establish the effect of sleep duration on the relationship between sleep apnea and cancer

did not study a specific type of tumor, histology or site (different cells can have a different sensitivity to the action of hypoxia); no valid marker of intermittent hypoxemia was used $[56,57,61,62 \bullet \bullet, 65]$, and the relatively limited power for subgroup analyses (e.g., non-obese, $<65$ years, etc.) due to the relatively small number of events.

\section{Studies in Progress}

At present, only one prospective study seeking to analyze the association between OSA and a specific type of tumor (skin melanoma) has been conducted by the Spanish Sleep Group. The study aims to verify whether the severity of OSA is associated with more aggressive behavior from this tumor. There was an initial pilot study in which 56 patients diagnosed with melanoma were subjected to respiratory polygraphy [66••]. The prevalence of OSA was high in this series; $60.7 \%$ of the patients presented an AHI $\geq 5$ and $14.3 \%$ had an AHI $\geq 30$. Moreover, the severity of OSA, whether measured by the AHI or the desaturation index (DI), was found to correlate with greater skin depth of the tumor and a higher rate of tumor growth. After adjusting for confounders, both the AHI and the DI proved to be independent predictors of faster melanoma growth. These findings have led to conduct a larger, multicenter study that will try to confirm these preliminary results.

\section{Conclusions}

Despite the growing interest in the possible association between sleep disorders and cancer, the scarcity of data and the limitations in the design of the studies undertaken so far make the latter little more than generators of hypotheses that produce more questions than answers, although they do encourage us to carry out further better designed studies to clarify this possible association (Table 3).

Acknowledgments Thanks to Dr. F. J. Nieto for reviewing this paper under his "Sleep Epidemiology" section published in volume 1, Issue 4 of 2015. This paper is funded by Fondo de Investigación Sanitaria (FIS) PI12 /01363, Beca SEPAR 058/2011, and Programa Beatriu de Pinós de la Generalitat de Catalunya (2010 BP_00238).

\section{Compliance with Ethical Standards}

Conflict of Interest Miguel Ángel Martínez-García, Francisco Campos-Rodríguez, and Isaac Almendros declare that they have no conflict of interest.

Human and Animal Rights and Informed Consent This article does not contain any studies with human or animal subjects performed by any of the authors.

\section{References}

Papers of particular interest, published recently, have been highlight as:

- Of importance

-. Of major importance

1. Blask DE. Melatonin, sleep disturbance and cancer risk. Sleep Med Rev. 2009;13:257-64.

2. Haus EL, Smolensky MH. Shift work and cancer risk: potential mechanistic roles of circadian disruption, light at night, and sleep deprivation. Sleep Med Rev. 2013;17:273-84.

3. Savvidis C, Koutsilieris M. Circadian rhythm disruption in cancer biology. Mol Med. 2012;18:1249-60.

4. Sliwinski T, Rozej W, Morawiec-Bajda A, Morawiec Z, Reiter R, Blasiak J. Protective action of melatonin against oxidative DNA damage: chemical inactivation versus base-excision repair. Mutat Res. 2007;634:220-7.

5. Hill SM, Frasch T, Xiang S, Yuan L, Duplessis T, Mao L. Molecular mechanisms of melatonin anticancer effects. Integr Cancer Ther. 2009;8:337-46.

6. Davis S, Mirick DK. Circadian disruption, shift work and the risk of cancer: a summary of the evidence and studies in Seattle. Cancer Causes Control. 2006;17:539-45.

7. Gery S, Komatsu N, Baldjyan L, Yu A, Koo D, Koeffler HP. The circadian gene Perl plays an important role in cell growth and DNA damage control in human cancer cells. Mol Med. 2006;22:375-82.

8. Yang X, Wood PA, Oh E-Y, Du-Quiton J, Ansell CM, Hrushesky WJM. Down regulation of circadian clock gene period 2 accelerates breast cancer growth by altering its daily growth rhythm. Breast Cancer Res Treat. 2009;117:423-31.

9. Taniguchi H, Fernández AF, Setién F, Ropero S, Ballestar E, Villanueva A, et al. Epigenetic inactivation of the circadian clock gene BMAL1 in hematologic malignancies. Cancer Res. 2009;69:8447-54.

10. Tokunaga H, Takebayashi Y, Utsunomiya H, Akahira J-I, Higashimoto M, Mashiko M, et al. Clinicopathological significance of circadian rhythm-related gene expression levels in patients with epithelial ovarian cancer. Acta Obstet Gynecol Scand. 2008;87:1060-70.

11. Barceló A, Barbé F. Estrés oxidativo y síndrome de apneashipopneas del sueño. Arch Bronconeumol. 2005;41:393-9.

12. Yamauchi M, Nakano H, Maekana J, Okamoto Y, Ohnishi T, Kimura H. Oxidative stress in obstructive sleep apnea. Chest. 2005;127:1674-9.

13. Mc Nicholas WT. Obstructive sleep apnea and inflammation. Prog Cardiovasc Dis. 2009;51:392-9. 
14. Semenza GL. Oxygen sensing, homeostasis, and disease. N Engl J Med. 2011;365:537-47. One of the best reviews in the scientific literature on oxygen sensing in diseases.

15. Vaupel P. The role of hypoxia-induced factors in tumor progression. Oncologist. 2004;9:10-7.

16. Semenza GL. Hypoxia-inducible factors: mediators of cancer progression and targets for cancer therapy. Trends Pharmacol Sci. 2012;33:207-14.

17. Palazon A, Aragonés J, Morales-Kastresana A, De Landezuri MO, Melero I. Molecular pathways: hypoxia response in immune cells fighting or promoting cancer. Clin Cancer Res. 2012;18:1207-13.

18. Toffoli S, Michiels C. Intermittent hypoxia is a key regulator of cancer cell and endothelial cell interplay in tumours. FEBS J. 2008;275:2991-3002.

19. Rofstad EK, Gaustad JV, Egeland TA, Mathiesen B, Galappathi K. Tumors exposed to acute cyclic hypoxic stress show enhanced angiogenesis, perfusion and metastatic dissemination. Int $\mathrm{J}$ Cancer. 2010;127:1535-46.

20. Martinive P, Defresne F, Bouzin C, et al. Preconditioning of the tumor vasculature and tumor cells by intermittent hypoxia: implications for anticancer therapies. Cancer Res. 2006;66:11736-44.

21. Eubank T, Sherwani S, Peters S, Gross A, Evans R, Magaland UJ. Intermittent hypoxia augments melanoma tumor metastases in a mouse model of sleep apnea. Am J Respir Crit Care Med. 2013; 187:A2302.

22. Mills PJ, Natarajan L, Von Kanel R, Ancoli-Israel S, Dimsdale JE. Diurnal variability of C-reactive protein in obstructive sleep apnea. Sleep Breath. 2009;13:415-20.

23. Xie X, Pan L, Ren D, Du C, Guo Y. Effects of continuous positive airway pressure therapy on systemic inflammation in obstructive sleep apnea: a meta-analysis. Sleep Med. 2013;14:1139-50.

24. Punjabi NM. The epidemiology of adult obstructive sleep apnea. Proc Am Thorac Soc. 2008;5:136-43.

25. Brandon EL, Gu JW, Cantwell L, He Z, Wallace G, Hall JE. Obesity promotes melanoma tumor growth role of leptin. Cancer Biol Ther. 2009;8:1871-9.

26. Basen-Engquist K, Chang M. Obesity and cancer risk: recent review and evidence. Curr Oncol Rep. 2011;13:71-6.

27. Calle EE, Rodriguez C, Walker-Thurmond K, Thun MJ. Overweight, obesity, and mortality from cancer in a prospectively studied cohort of U.S. adults. N Engl J Med. 2003;348:1625-38.

28. Flegal KM, Kit BK, Orpana H, Graubard BI. Association of allcause mortality with overweight and obesity using standard body mass index categories: a systematic review and meta-analysis. JAMA. 2013;309:71-82.

29. Lewis C, Murdoch C. Macrophage responses to hypoxia: implications for tumor progression and anti-cancer therapies. Am J Pathol. 2005;167(3):627-35.

30. Gileles-Hillel A, Almendros I, Khalyfa A, Zhang SX, Wang Y, Gozal D. Early intermittent hypoxia induces proatherogenic changes in aortic wall macrophages in a murine model of obstructive sleep apnea. Am J Respir Crit Care Med. 2014;190(8):958-61.

31. Carreras A, Zhang SX, Almendros I, et al. Resveratrol Attenuates Intermittent Hypoxia-Induced Macrophage Migration to Visceral White Adipose Tissue and Insulin Resistance in Male Mice. Endocrinology 2014; en20141706.

32. Van OE, Laoui D, Keirsse J, Van Ginderachter JA. Hypoxia and tumor-associated macrophages: a deadly alliance in support of tumor progression. Oncoimmunology. 2014;3(1), e27561.

33. Wagner M, Bjerkvig R, Wiig H, et al. Inflamed tumor-associated adipose tissue is a depot for macrophages that stimulate tumor growth and angiogenesis. Angiogenesis. 2012;15(3):481-95.

34. Almendros I, Gileles-Hillel A, Khalyfa A, et al. Adipose tissue macrophage polarization by intermittent hypoxia in a mouse model of OSA: Effect of tumor microenvironment. Cancer Lett 2015.
35. Almendros I, Wang Y, Becker L, et al. Intermittent hypoxia-induced changes in tumor-associated macrophages and tumor malignancy in a mouse model of sleep apnea. Am J Respir Crit Care Med. 2014;189(5):593-601.

36. Shah PN, Mhatre MC, Kothari LS. Effect of melatonin on mammary carcinogenesis in intact and pinealectomized rats in varying photoperiods. Cancer Res. 1984;44:3403-7.

37. Blask DE. Melatonin-depleted blood from premenopausal women exposed to light at night stimulates growth of human breast cancer xenografts in nude rats. Cancer Res. 2005;65:11174-84.

38. Filipski E, Delaunay F, King VM, Wu M-W, Claustrat B, GréchezCassiau A, et al. Effects of chronic jet lag on tumor progression in mice. Cancer Res. 2004;64:7879-85.

39. Filipski E, King VM, Li X, Granda TG, Mormont M-C, Liu X, et al. Host circadian clock as a control point in tumor progression. J Natl Cancer Inst. 2002;94:690-7.

40. Almendros I, Montserrat JM, Torres M, et al. Intermittent hypoxia increases melanoma metastasis to the lung in a mouse model of sleep apnea. Respir Physiol Neurobiol. 2013;186(3):303-7.

41.• Almendros I, Montserrat JM, Ramirez J, et al. Intermittent hypoxia enhances cancer progression in a mouse model of sleep apnoea. Eur Respir J. 2012;39(1):215-7. First animal study that finds a positive relationship between the severity of induced intermittent hypoxia in a mouse model and the growth of tumor.

42. Almendros I, Montserrat JM, Torres M, et al. Obesity and intermittent hypoxia increase tumor growth in a mouse model of sleep apnea. Sleep Med. 2012;13(10):1254-60.

43. Hakim F, Wang Y, Zhang SX, et al. Fragmented sleep accelerates tumor growth and progression through recruitment of tumorassociated macrophages and TLR4 signaling. Cancer Res. 2014;74(5):1329-37.

44. Straif K, Baan R, Grosse Y, Secretan B, El Ghissassi F, Bouvard V, et al. Carcinogenicity of shift-work, painting, and fire-fighting. Lancet Oncol. 2007;8:1065-6.

45. Schernhammer ES, Kroenke CH, Laden F, Hankinson SE. Night work and risk of breast cancer. Epidemiology. 2006;17:108-11.

46. Viswanathan AN, Hankinson SE, Schernhammer ES. Night shift work and the risk of endometrial cancer. Cancer Res. 2007;67: 10618-22

47. Hansen J. Increased breast cancer risk among women who work predominantly at night. Epidemiology. 2001;12:74-7.

48. Verkasalo PK, Pukkala E, Stevens RG, Ojamo M, Rudanko SL. Inverse association between breast cancer incidence and degree of visual impairment in Finland. Br J Cancer. 1999;80:1459-60.

49. Kubo T. Prospective cohort study of the risk of prostate cancer among rotating-shift workers: findings from the Japan collaborative cohort study. Am J Epidemiol. 2006;164:549-55.

50. Verkasalo PK. Sleep duration and breast cancer: a prospective cohort study. Cancer Res. 2005;65:9595-600.

51. Kakizaki M, Kuriyama S, Sone T, Ohmori-Matsuda K, Hozawa A, Nakaya N, et al. Sleep duration and the risk of breast cancer: the Ohsaki cohort study. Br J Cancer. 2008;99:1502-5.

52. Wu AH, Wang R, Koh W-P, Stanczyk FZ, Lee H-P, Yu MC. Sleep duration, melatonin and breast cancer among Chinese women in Singapore. Carcinogenesis. 2008;29:1244-8.

53. Pinheiro SP. A prospective study on habitual duration of sleep and incidence of breast cancer in a large cohort of women. Cancer Res. 2006;66:5521-5.

54. Vogtmann E, Levitan EB, Hale L, Shikany JM, Shah NA, Endeshaw Y, et al. Association between sleep and breast cancer incidence among postmenopausal women in the Women's Health Initiative. SLEEP. 2013;36:1437-44.

55. von Ruesten A, Weikert C, Fietze I, Boeing H. Association of sleep duration with chronic diseases in the European Prospective Investigation into Cancer and Nutrition (EPIC)-Postdam study. PLos One 2012;7(1):e30972 
56. McElroy JA, Newcomb PA, Titus-Ernstoff L, Trentham-Dietz A, Hampton JM, Egan KM. Duration of sleep and breast cancer risk in a large population-based case-control study. J Sleep Res. 2006;15:241-9.

57. Zhang X, Giovannucci EL, Wu K, Gao X, Hu F, Ogino S, et al. Associations of self-reported sleep duration and snoring with colorectal cancer risk in men and women. Sleep. 2013;36:681-8.

58.• Nieto FJ, Peppard PE, Young T, Finn L, Hla KM, Farre R. Sleepdisordered breathing and cancer mortality: results from the Wisconsin sleep cohort study. Am J Respir Crit Care Med. 2012;186:190 - 4. First based-populatio study that finds a positive relationship between the severity of obstructive sleep apnea, especially the severity of nocturnal hypoxia, and the increase mortality of all types of cancer.

59. Marshall NS, Wong KKH, Cullen SRJ, Knuiman MW, Grunstein RR. Sleep apnea and 20-year follow-up for all-cause mortality, stroke, and cancer incidence and mortality in the Busselton health study cohort. J Clin Sleep Med. 2014;10:355-62.

60. Chang W-P, Liu M-E, Chang W-C, et al. Sleep apnea and the subsequent risk of breast cancer in women: a nationwide populationbased cohort study. Sleep Med. 2014;15:1016-20.

61. Chen J-C, Hwang J-H. Sleep apnea increased incidence of primary central nervous system cancers: a nationwide cohort study. Sleep Med. 2014;15:749-54.
62.• Campos-Rodriguez F, Martinez-Garcia MA, Martinez M, et al. Association between obstructive sleep apnea and cancer incidence in a large multicenter Spanish cohort. Am J Respir Crit Care Med. 2013;187:99-105. First clinical study that finds a positive relationship between the severity of obstructive sleep apnea, especially the severity of nocturnal hypoxia, and the increase incidence of all types of cancer.

63. Martínez-García MA, Campos-Rodriguez F, Durán-Cantolla J, et al. Obstructive sleep apnea is associated with cancer mortality in younger patients. Sleep Med. 2014;15:742-8.

64. Kendzerska T, Leung RS, Hawker G, Tomlinson G, Gershon AS. Obstructive sleep apnea and the prevalence and incidence of cancer. CMAJ. 2014;186:985-92.

65. Christensen AS, Clark A, Salo P, Nymann P, Lange P, Prescott E, et al. Symptoms of sleep disordered breathing and risk of cancer: a prospective cohort study. Sleep. 2013;36:1429-35.

66.• Martínez-García M-Á, Martorell-Calatayud A, Nagore E, et al. Association between sleep disordered breathing and aggressiveness markers of malignant cutaneous melanoma. Eur Respir J. 2014;43:1661-8. First prospective study in cancerous patients that analyse the relationship between the number and severity of sleep-disordered breathing and aggresiveness of melanoma. 